Danielle Constância Felício Macedo ${ }^{\mathrm{a}}$ (iD) http://orcid.org/0000-0003-0231-4102

Priscilla de Oliveira Martins-Silva ${ }^{\mathrm{b}}$ (iD) https://orcid.org/0000-0002-2922-6607

Adriana Judith Esteves Fantini ${ }^{\mathrm{a}}$ (iD) https://orcid.org/0000-0002-4606-1664

Cíntia Yuri Soga Bomfim Machado ${ }^{c}$ (iD) https://orcid.org/0000-0002-3871-934X

Fabíola de Oliveira Lima ${ }^{\mathrm{a}}$

(iD) https://orcid.org/0000-0003-4713-747X

Geraldo Alves Lacerda ${ }^{a}$

(iD) https://orcid.org/0000-0002-1748-1408

Mariana Angélica Peixoto de Souza (iD) https://orcid.org/0000-0002-1096-7085

Patrícia de Rizzo Toledo ${ }^{a}$

(iD) https://orcid.org/0000-0003-3852-8911

Suzan Caroline Such Ribeiro ${ }^{\mathrm{a}}$

(iD) https://orcid.org/0000-0002-0982-6357

a Universidade Federal de Minas Gerais (UFMG), Pró-Reitoria de Recursos Humanos, Departamento de Atenção à Saúde do Trabalhador. Belo Horizonte, MG, Brasil.

${ }^{\mathrm{b}}$ Universidade Federal do Espirito Santo (UFES), Departamento de Administração, Programa de PósGraduação em Administração. Vitória, ES, Brasil.

'Instituto Federal de Educação Ciência e Tecnologia de São Paulo, Campus São Carlos, Coordenadoria Sociopedagógica. São Carlos, SP, Brasil.

Contato:

Priscilla de Oliveira Martins-Silva

E-mail:

priscillamartinssilva@gmail.com

Os autores declaram que o trabalho não foi subvencionado e que não há conflitos de interesses.

Os autores informam que o trabalho não foi apresentado em evento científico e que foi baseado na tese de doutorado Reinserção no trabalho: estudo do fenômeno em uma Instituição Federal de Ensino Superior, de Danielle Constância Felício Macedo, defendida em 2019 no Programa de Pós-Graduação em Psicologia da Universidade Federal do Espírito Santo.

Recebido: 09/02/2018

Revisado: 20/12/2018

Aprovado: 25/01/2019

\section{Implantação de ações de reabilitação funcional em uma instituição federal de ensino superior}

\author{
Implementation of functional rehabilitation \\ measures in a federal university
}

\begin{abstract}
Resumo
Introdução: a reabilitação funcional (RF) se apresenta como um elo entre os cuidados de saúde e as demandas do contexto laboral e envolve, necessariamente, intervenções no contexto de trabalho. Objetivo: apresentar a implantação de ações de RF em uma instituição federal de ensino superior (Ifes) da região Sudeste. Métodos: são descritos o processo de definição teóricometodológico, a problematização dos princípios versados na legislação vigente, os critérios e procedimentos aplicados na RF, as possibilidades e os limites do contexto institucional. São analisados os resultados do programa no primeiro ano de implantação da RF, por meio de avaliação de casos de trabalhadores reinseridos. Resultados: as ações de RF foram oferecidas para nove casos-piloto direcionados para a equipe e distribuídas entre seus membros. No primeiro ano do programa, 2016, após a implantação de ações de RF, foi observada redução de $37 \%$ no percentual médio do número de dias de afastamento do trabalho, comparada aos afastamentos do ano anterior. Conclusão: é importante elaborar diretrizes políticas que melhor definam o escopo conceitual e epistemológico da RF, refletidas na legislação, e que apresentem possibilidades teóricometodológicas para viabilizar a implantação de serviços de RF nas Ifes.
\end{abstract}

Palavras-chave: setor público, retorno ao trabalho, reabilitação, saúde do trabalhador.

\begin{abstract}
Introduction: functional rehabilitation (FR) is a link between healthcare and work demands, and necessarily leads to intervention in the working context. Objective: to present the implementation of $F R$ measures in a federal university from the Southeast region of Brazil. Methods: we described the FR theoreticalmethodological definition, the principles included in the current legislation, the criteria and procedures applied to FR as well as its institutional possibilities and limitations. We evaluated the results of the first year of FR implementation, 2016, by analyzing cases of returning to work. Results: FR measures were provided to nine pilot cases. After the first year, there was a reduction of $37 \%$ in sick leave, compared to the previous year. Conclusion: it is important to formulate policies and legislation that better define the FR conceptual/epistemological scope and present theoretical/methodological framework for enabling the implementation of FR services in federal universities.
\end{abstract}

Keywords: public sector; return to work; rehabilitation; occupational health. 


\section{Introdução}

Os determinantes do contexto laboral que podem afetar a saúde de servidores públicos vivenciados no exercício de suas funções são de natureza diversa. Dentre eles, podem-se citar situações de precarização do trabalho, como privatizações ou terceirizações ${ }^{1,2}$; condições inadequadas de trabalho ${ }^{2,3}$; dedicação do servidor a ações e projetos com resultados e futuro incertos, decorrentes da instabilidade orçamentária, mudanças políticas ou de gestão ${ }^{3-5}$; acúmulo ou sobrecarga de funções, entre outras fragilidades da gestão pública ${ }^{2-5}$; falta de reconhecimento da função social e importância do servidor público ${ }^{2,3}$ e estereótipo do servidor associado à morosidade e à baixa qualidade dos serviços prestados ${ }^{1-3}$.

A literatura tem mostrado os impactos desses determinantes na saúde de servidores de diversos seguimentos, decorrentes da problematização de fatores ligados a processos de sofrimento no trabalho ${ }^{2}$, aos índices de afastamento do trabalho $\mathrm{o}^{6,7}$, aos riscos psicossociais $^{8}$ e aos indicadores de qualidade de vida ${ }^{9-11}$.

No que tange à saúde de servidores públicos federais (SPF), destacam-se os esforços para a consolidação de estratégias de promoção, prevenção e acompanhamento da saúde desses trabalhadores com a implantação do Subsistema Integrado de Atenção à Saúde do Servidor Público Federal (Siass) em $2009^{12}$. O Siass prevê intervenções que abrangem o processo de adoecimento do servidor, tanto no aspecto individual quanto nas relações coletivas no ambiente de trabalho, cujo objetivo é a manutenção da funcionalidade laboral do servidor ativo ou em processo de retorno ao trabalho ${ }^{12}$.

Nesse sentido, profissionais do Serviço de Saúde do Trabalhador (SST) de uma instituição federal de ensino superior (Ifes) da região Sudeste iniciaram, em 2016, a implementação de ações de reabilitação funcional (RF) na instituição. O Quadro 1 apresenta a legislação vigente sobre as ações de $\mathrm{RF}$ voltadas para o SPF. A RF, neste trabalho, consiste em um processo terapêutico realizado no âmbito laboral dos SPF, voltado a oferecer suporte técnico para o manejo das questões de saúde e da funcionalidade do servidor relacionadas ao contexto de trabalho. Para se referir aos processos de retorno ao trabalho envolvendo limitações físicas e/ou mentais, a literatura ${ }^{13-15}$ e a legislação ${ }^{16,17}$ utilizam termos como "reinserção", "reabilitação", "readaptação" e "reintegração", muitas vezes empregados como sinônimos.

A proposta de RF se diferencia de programas de promoção à saúde e de qualidade de vida no trabalho ${ }^{18-21}$ por não predefinir recurso, técnica, frequência ou duração da intervenção. Somente após a análise da demanda e considerando os recursos disponíveis define-se um plano de trabalho individual para o servidor em RF. Esse processo envolve o acompanhamento do servidor com alguma condição de saúde que altere sua funcionalidade no contexto de trabalho de modo longitudinal, durante um período de tempo finito, até que os recursos disponíveis para a demanda identificada tenham sido explorados e seus resultados avaliados.

Quadro 1 Legislação sobre reabilitação funcional no Serviço Público Federal

Manual de Perícia Oficial

em Saúde do Subsistema

Integrado de Atenção à Saúde

do Servidor Público Federal ${ }^{16}$

\begin{tabular}{|l|l|}
\hline & $\begin{array}{l}\text { Entende-se por reabilitação um conjunto de ações e intervenções que visam a melhorar a } \\
\text { reestruturação da autonomia da pessoa nas suas dimensões física, mental, social e afetiva, } \\
\text { integrando-a nos diferentes espaços da sociedade. A reabilitação tem como objetivo diminuir ou } \\
\text { eliminar as limitações sofridas pelo servidor para o exercício de suas atividades laborais e valorizar as } \\
\text { capacidades e competências. } \\
\text { I- Estimular a criação de grupos de readaptação, ressocialização, apoio terapêutico e reinserção nos } \\
\text { Princípios, Diretrizes e Ações } \\
\text { em Saúde Mental }{ }^{17} \\
\text { locais de trabalho, conforme a realidade, como forma de lidar com as demandas de reabilitação; } \\
\text { Il prover recursos e estratégias terapêuticas que valorizem as habilidades, competências e talentos } \\
\text { III- propiciar a realização de intervenções terapêuticas não medicalizantes para estabilização de } \\
\text { quadros clínicos apresentados pelos servidores; } \\
\text { IV- orientar e capacitar os servidores para exercerem atividades compatíveis com sua capacidade } \\
\text { laborativa e seus interesses; e } \\
\text { V- sensibilizar gestores para o acolhimento dos servidores no retorno ao trabalho. }\end{array}$ \\
\hline
\end{tabular}
Processo de duração limitada, com objetivo definido, destinado a recuperar pessoa com incapacidade adquirida para alcançar níveis físicos, mentais e funcionais que possibilitem seu retorno ao trabalho. 
No Brasil, as taxas de afastamento do trabalho por motivo de doença de servidores das Ifes seguem alarmantes. Dados do Portal da Transparência do Governo Federal apontam que no ano de 2016 foram realizadas cerca de 56 mil perícias médicas entre servidores dessas instituições, gerando o total de 1,6 milhão de dias de afastamento ${ }^{22}$.

A Licença para Tratamento de Saúde (LTS) e a ocorrência de alterações na funcionalidade do servidor no seu processo de adoecimento, por vezes, acarretam desafios para seu retorno ao trabalho e para a (re)adaptação às antigas ou às novas rotinas laborais ${ }^{13-15}$. Vivian ${ }^{23}$ realizou um estudo qualitativo com servidores readaptados e chefias, evidenciando: a ausência de equipe multidisciplinar institucional; o fato de o próprio trabalhador procurar o posto de trabalho após o laudo de readaptação; o sentimento do servidor de incompetência e culpa; e situações de discriminação. Santos ${ }^{24}$ analisou um caso de readaptação e concluiu pela importância dos processos de acolhimento e da configuração de espaços para escuta entre os sujeitos que compartilham aspectos de um mesmo processo de trabalho. Esses resultados indicam a relevância dos processos de RF para que o trabalhador possa ser reinserido de acordo com sua condição de saúde e funcionalidade ${ }^{24}$.

O cenário da RF no Brasil revela a premência de se efetivar uma mudança de paradigma na compreensão da funcionalidade e incapacidade do servidor, visando a fortalecer as políticas públicas voltadas para acolher indivíduos com alguma incapacidade para o trabalho decorrente de questões de saúde ${ }^{25}$. Paralelo a isso, tal cenário aponta ainda para a importância de considerar a atuação no ambiente de trabalho, abordando as múltiplas variáveis envolvidas na saúde do trabalhador, de modo a adequar o trabalho a quem o executa ${ }^{26,27}$, prevenir riscos de acidentes e agravos à saúde, atuando, principalmente, na promoção à saúde.

A proposta deste trabalho é inovadora à medida que apresenta um relato descritivo de reflexões e vivências de uma equipe de RF na busca por fundamentos e métodos para a consolidação de suas ações, dado que a legislação vigente não é elucidativa. Ressalta-se, no entanto, que o conjunto das teorias, dos métodos e das ações terapêuticas que compõe este trabalho são amplamente debatidos na literatura, quais sejam, a presença de uma equipe interdisciplinar ${ }^{28,29}$, a ergonomia da atividade (EA) ${ }^{26,27}$, a ergologia ${ }^{30-33}$ e o Modelo de Funcionalidade e Incapacidade da Organização Mundial de Saúde (OMS) ${ }^{34-36}$. Este relato se destaca por explorar interseções entre o conhecimento de diferentes categorias profissionais, modelos de saúde e as diversas abordagens em saúde do trabalhador, buscando lapidar diretrizes para as práticas e implantação da RF.

O objetivo deste relato é apresentar a experiência da implantação de ações de reabilitação funcional realizadas pela equipe interdisciplinar do Serviço de Saúde do Trabalhador da instituição federal de ensino superior onde foi realizada.

\section{Métodos}

Descrevem-se: a problematização dos princípios versados na legislação diante das demandas dos servidores; as possibilidades e limites do contexto institucional; a busca por referenciais teóricos e por um modelo de saúde norteadores do trabalho; e os principais desafios e reflexões da equipe que permearam o processo de implantação da RF.

Este trabalho está vinculado a um projeto de pesquisa aprovado pelo Comitê de Ética da Universidade Federal do Espírito Santo, sob parecer $\mathrm{n}^{\circ}$ 1.970.746, CAAE 63023216.9.0000.5542.

\section{Desenvolvimento da experiência}

A equipe responsável pela implementação da RF definiu como pilares de sua atuação: a interdisciplinaridade, a intersetorialidade e o interesse pela ação no trabalho em que o protagonismo dos trabalhadores seja contemplado. Em março de 2016, a implantação teve início com quatro membros e, ao final desse ano, expandiu para sete: três psicólogas, duas fisioterapeutas, uma terapeuta ocupacional e um psiquiatra. Todos desenvolviam outras atribuições no SST, sendo a carga horária dedicada à RF restrita.

O projeto tinha como objetivos: (a) incentivar o desenvolvimento de ações interdisciplinares e intersetoriais em promoção à saúde pautadas no protagonismo do trabalhador nos processos de análise, reflexão e transformação dos contextos de trabalho na universidade; (b) desenvolver ações de prevenção de agravos para servidores em processo de adoecimento e fragilidades na saúde já adquiridos; (c) estabelecer aproximações com o trabalhador e com o trabalho como prerrogativa para a intervenção; (d) favorecer a comunicação e a articulação de ações em saúde do trabalhador. 
Em busca de diretrizes para a reabilitação funcional: preocupações epistemológicas, conceituais e teóricas

A equipe debateu sobre o escopo da ação de RF, especificamente acerca da natureza de seu aspecto clínico. Considerando a atuação de profissionais na rede de saúde pública e suplementar (RSS), refletiu-se qual atribuição da equipe complementaria as ações da RSS. Qual seria o papel do profissional de saúde do trabalhador dentro da instituição, especificamente na RF? Como estruturar o trabalho da equipe para complementar as ações de outros profissionais da RSS envolvidos no cuidado à saúde do servidor? Como sedimentar a RF, como uma estratégia de saúde do trabalhador, com a mobilização e a atuação em rede junto a outros setores da própria instituição e aos profissionais da RSS?

Considerou-se necessário definir a expressão RF, ancorada na legislação vigente, apesar de seu conteúdo genérico. O uso isolado dos termos "reinserção", "reabilitação", "readaptação" e "reintegração" poderia gerar equívocos acerca da questão. Assim, a equipe deliberou pela importância de se apropriar da terminologia e de colaborar com a literatura do campo, elaborando considerações epistemológicas que corroborem a especificidade das ações de RF.

Constatou-se a existência, dentro da própria equipe e no diálogo com outros atores institucionais, de múltiplas conotações de RF, tendo sido debatida a necessidade de delinear uma diretriz para significar as práticas de RF. Observou-se, por exemplo, o entendimento da terminologia "reabilitação" como referente a técnicas aplicadas por diferentes especialidades para a recuperação do indivíduo após alguma lesão ou processo de adoecimento. O termo "funcional" foi ainda vinculado ao contexto de práticas clínicas assistenciais na RSS.

O desenvolvimento das ações da equipe ocorreu na interface entre as abordagens clínicas das disciplinas que compunham a equipe. Os profissionais adotaram o desafio cotidiano de estabelecer ações cujas bases e diretrizes fossem congruentes em meio à diversidade de técnicas e de recursos terapêuticos disponíveis, visando a consolidar fundamentos transversais às práticas de RF.

Discutiu-se a importância de conectar as práticas da equipe a um modelo de saúde que apresentasse consonância com a proposta da RF e a necessidade de alinhavar a RF a princípios e pressupostos do campo da saúde do trabalhador. Reflexões foram iniciadas no intuito de diferenciar a $\mathrm{RF}$ de procedimentos assistenciais realizados em contextos clínicos ambulatoriais ou hospitalares. Isto é, não se tratava de ações típicas de assistência realizadas em estabelecimentos de saúde, já que era preciso considerar aspectos da atividade de trabalho.

Assim, chegou-se ao entendimento de que a RF consiste em um processo terapêutico realizado no âmbito laboral dos servidores, cujas ações oferecem suporte técnico no manejo das questões de saúde e da funcionalidade do servidor relacionadas ao contexto de trabalho; atua na interseção do processo saúde-doença com os fatores ligados à atividade de trabalho. Para tanto, recorreu-se a abordagens teórico-metodológicas no campo da saúde do trabalhador que congregam como premissa básica o interesse pela ação no trabalho ${ }^{28}$, sendo a EA e a ergologia os referenciais explorados num primeiro momento; e ao Modelo de Funcionalidade e Incapacidade da $\mathrm{OMS}^{35,36}$, que propõe a Classificação Internacional de Funcionalidade, Incapacidade e Saúde (CIF) ${ }^{34,35}$.

Assim, a atuação da equipe diante da referida interseção de saberes requisitada pela RF consistiu em um processo dinâmico cuja construção se deu e se renovou no cotidiano do trabalho. Tal processo não esteve limitado apenas ao apresentado neste relato, tendo sido selecionadas as principais reflexões, ações e decisões impelidas pela prática e pelos desafios vivenciados na estruturação do projeto.

\section{O primeiro contato com o campo: problematizando a} demanda de reabilitação funcional e seus contextos

A equipe teve contato com queixas de trabalhadores e suas chefias, tendo iniciado sua atuação de modo exploratório, identificando a existência de demandas circunscritas ao que a legislação define como RF. Buscou-se delinear especificamente quais as demandas para RF, considerando: as normativas sobre a RF; a análise de queixas relacionadas ao trabalho de servidores no decurso de processos de adoecimento atendidas no SST (muitas vezes ligadas a situações de conflito no ambiente laboral); e a existência de casos em situação cronificada ou com baixa resolutividade apesar dos esforços dos dispositivos institucionais acionados.

Constatou-se que os processos de adoecimento que acarretaram mudanças na condição de saúde e na funcionalidade de trabalhadores eram situações potenciais para a atuação da RF, identificadas em circunstâncias como: avaliação da Perícia Oficial em Saúde (POS), em que o trabalhador poderia ser 
considerado capaz para o trabalho, embora com restrições laborais (temporárias ou permanentes); atendimento a trabalhadores que buscavam o serviço de assistência no SST e sinalizavam a existência de problemas no trabalho em função de sua condição de saúde e funcionalidade; e trabalhadores que procuravam o setor de Recursos Humanos (RH) com queixas de dificuldades no trabalho, que apresentavam interface com questões de saúde.

Via de regra, essas situações de queixas relacionadas ao trabalho geravam intervenção de diversos atores institucionais, envolvendo tomadas de decisões complexas, principalmente no momento do retorno ao trabalho após LTS, tais como decidir se: o trabalhador seria direcionado para o setor de origem ou para outro setor? O retorno ocorreria para as mesmas ou novas atividades de trabalho? Seria necessária a realização de adaptações que favoreçam a segurança e o desempenho do servidor, valorizando suas competências e capacidades remanescentes? Frequentemente, observou-se que tais decisões e intervenções eram problemáticas e desafiadoras para a instituição.

A equipe iniciou sua abordagem em alguns casos nos quais intervenções anteriores não envolveram a perspectiva da RF e constatou-se, pelo histórico de adoecimento do servidor, que houve crescimento do número de queixas, do agravamento das questões de saúde e/ou a ocorrência de conflitos interpessoais no ambiente de trabalho. Essas situações sinalizaram a premência de que as diversas instâncias, que atuavam com o servidor, estabelecessem ações parceiras, complementares e resolutivas.

No contexto observado, é importante salientar pontos críticos que desafiam as práticas de RF: (a) geralmente, as restrições laborais se referem a movimentos corporais, procedimentos e atividades de trabalho que o trabalhador deve evitar desempenhar, e o cumprimento dessas restrições pode não ser alcançado na definição do que, de fato, o trabalhador executará no rol das atribuições de seu cargo; (b) percebe-se a ocorrência frequente de condutas culpabilizantes e preconceituosas, bem como a desvalorização do trabalhador em seu setor e sua desmotivação diante dos limites na execução modificada das atividades laborais e situações de sobrecarga de outros colegas de trabalho; (c) o trabalhador adoecido, por vezes, não se reconhece ou não se sente reconhecido em seu grupo de trabalho, dando lugar ao sentimento de não pertencimento e de invisibilidade ${ }^{37}$. Ademais, a depender da atividade-fim e dos riscos presentes para o trabalhador ou para terceiros, é ainda mais imprescindível que a $R F$ viabilize um planejamento seguro da execução das atividades e contemple o ajustamento global das atividades de trabalho do setor.

\section{Definição dos procedimentos de reabilitação funcional ao longo do primeiro ano de implantação}

As ações de RF foram oferecidas em nove casos-piloto direcionados para a equipe antes mesmo que esta pudesse definir critérios de encaminhamento pautados em indicadores epidemiológicos ou estatísticas de adoecimento e afastamento dos trabalhadores da Ifes. Assim, inicialmente, os casos acolhidos para a RF foram encaminhados pela Perícia Oficial em Saúde, do Serviço de Saúde do Trabalhador, e pelo setor de RH da Instituição, sendo que identificou-se somente a posteriori que o encaminhamento fora pautado na complexidade clínica e necessidade de manejo das questões de saúde e da funcionalidade do trabalhador relacionadas ao contexto de trabalho.

Esses casos foram distribuídos entre os membros da equipe, para que um profissional atuasse como a principal referência técnica da RF. Para elucidar a demanda em cada caso, foram realizadas: entrevista individual com o trabalhador; análise de prontuário médico e documentos; e visitas ao ambiente de trabalho para observação do servidor em atividade e das características do trabalho prescrito e real, com foco no desempenho do servidor mediante suas atribuições. A partir de então, para cada caso, foi elaborado um Plano de Trabalho em RF, contendo o planejamento das ações nos primeiros 90 dias de acompanhamento, previamente discutido e aprovado em reunião de equipe e, posteriormente, validado com o servidor. Esse plano foi ajustado no decurso do acompanhamento do servidor, considerando a ocorrência de novos fatos ou informações relevantes, sendo a duração total do acompanhamento de cada servidor variável.

No Quadro 2 encontra-se a estruturação metodológica elaborada no primeiro ano de atuação da equipe. Ressalta-se que a construção e a consolidação dessa proposta têm se dado paralelamente ao acolhimento de novos casos e ao delineamento das intervenções. 
Quadro 2 Critérios, objetivos e procedimentos da reabilitação funcional*

\begin{tabular}{|c|c|c|}
\hline \multirow{3}{*}{$\begin{array}{l}\text { Critérios de } \\
\text { encaminhamento }(C)\end{array}$} & $\mathrm{C} 1^{\text {楼 }}$ & $\begin{array}{l}\text { O quadro de saúde do servidor interfere de forma significativa no seu } \\
\text { desempenho laboral ou o processo de adoecimento do servidor está } \\
\text { relacionado ao contexto de trabalho. }\end{array}$ \\
\hline & $\mathrm{C} 2$ & $\begin{array}{l}\text { Necessidade de intervenção no ambiente/contexto de trabalho com foco na } \\
\text { saúde do trabalhador. }\end{array}$ \\
\hline & $\mathrm{C} 3$ & $\begin{array}{l}\text { Necessidade de preparação para o retorno ao trabalho após licença para } \\
\text { tratamento de saúde. }\end{array}$ \\
\hline \multirow{7}{*}{ Objetivos (O) } & 01 & $\begin{array}{l}\text { Dar suporte no manejo das questões de saúde e da funcionalidade do } \\
\text { trabalhador relacionadas ao contexto de trabalho. }\end{array}$ \\
\hline & $\mathrm{O} 2$ & $\begin{array}{l}\text { Viabilizar in loco o cumprimento de restrições da atividade laboral, quando } \\
\text { houver. }\end{array}$ \\
\hline & $\mathrm{O} 3$ & $\begin{array}{l}\text { Oferecer suporte técnico e sensibilizar colegas e gestores para o } \\
\text { acolhimento dos servidores em processo de reabilitação. }\end{array}$ \\
\hline & $\mathrm{O} 4$ & $\begin{array}{l}\text { Fomentar nos servidores (servidor/chefia/colegas) o desenvolvimento de } \\
\text { alternativas para lidar com conflitos e com as variabilidades do trabalho. }\end{array}$ \\
\hline & $\mathrm{O5}$ & $\begin{array}{l}\text { Abordar aspectos motivacionais relacionados ao trabalho e valorizar as } \\
\text { capacidades e competências. }\end{array}$ \\
\hline & O6 & $\begin{array}{l}\text { Auxiliar no desenvolvimento de habilidades que favoreçam o } \\
\text { relacionamento interpessoal no trabalho. }\end{array}$ \\
\hline & 07 & $\begin{array}{l}\text { Favorecer o autocuidado e autonomia no processo saúde-doença } \\
\text { (orientações e educação em saúde). }\end{array}$ \\
\hline \multirow{6}{*}{$\begin{array}{l}\text { Estratégias de } \\
\text { intervenção******* (I) }\end{array}$} & $\mathrm{I}-1$ & Análise da atividade de trabalho. \\
\hline & $1-2$ & Acompanhamento do servidor in loco. \\
\hline & $1-3$ & $\begin{array}{l}\text { Prescrição/confecção de equipamentos/adaptações na atividade } \\
\text { de trabalho. }\end{array}$ \\
\hline & $\mathrm{I}-4$ & Intervenções individuais e grupais (trabalhador, chefia, colegas). \\
\hline & $1-5$ & $\begin{array}{l}\text { Mediação em saúde do trabalhador, no sentido de facilitar a interação e } \\
\text { o diálogo entre o trabalhador e demais interlocutores: colegas, gestores, } \\
\text { membros do SST e RH, entre outros. }\end{array}$ \\
\hline & $\mathrm{I}-6$ & Encaminhamentos para outros serviços internos e externos. \\
\hline \multirow{7}{*}{$\begin{array}{l}\text { Atribuições dos } \\
\text { técnicos de referência } \\
\text { em RF }\end{array}$} & \multicolumn{2}{|c|}{ Avaliar o servidor e o contexto de trabalho. } \\
\hline & \multicolumn{2}{|c|}{ Definir o plano de trabalho a partir da discussão com a equipe. } \\
\hline & \multicolumn{2}{|c|}{ Envolver os atores que forem necessários nesse processo. } \\
\hline & \multicolumn{2}{|c|}{ Incentivar que os trabalhadores envolvidos busquem ações direcionadas ao seu autocuidado. } \\
\hline & \multicolumn{2}{|c|}{$\begin{array}{l}\text { Realizar orientações gerais de promoção à saúde e prevenção de agravos, contextualizadas ao } \\
\text { histórico clínico do servidor. }\end{array}$} \\
\hline & \multicolumn{2}{|c|}{ Sensibilizar e monitorar sua apropriação aos tratamentos que lhe foram indicados. } \\
\hline & \multicolumn{2}{|c|}{$\begin{array}{l}\text { Realizar atividades correlatas às descritas com o objetivo de conduzir adequadamente as ações } \\
\text { de RF. }\end{array}$} \\
\hline \multirow{2}{*}{$\begin{array}{l}\text { Atribuições dos técnicos de } \\
\text { referência em RF específicas para } \\
\text { casos de servidores com laudos } \\
\text { restritivos }\end{array}$} & \multicolumn{2}{|c|}{ Favorecer o cumprimento do laudo e documentar no prontuário o andamento desse processo. } \\
\hline & \multicolumn{2}{|c|}{$\begin{array}{l}\text { Comunicar à perícia médica, para que as restrições laborais sejam reavaliadas no sentido de } \\
\text { se definir por sua manutenção, descontinuidade ou necessidade de alteração, na ocasião do } \\
\text { surgimento de novos e significativos elementos vinculados à condição de saúde do servidor ou a } \\
\text { suas atividades laborais. }\end{array}$} \\
\hline
\end{tabular}

* Definidos pela equipe interdisciplinar do Serviço de Saúde do Trabalhador no ano de implantação das ações de RF; ** os códigos apresentados nesta tabela são utilizados no texto deste manuscrito e na Tabela 3; ; ${ }^{*}$ quando pertinente, são realizadas em interlocução com outros atores institucionais, por exemplo, membros do setor de RH. 
A seguir são apresentados os dados referentes às intervenções realizadas em oito dos nove casos-piloto. O nono servidor foi acompanhado no período de LTS, mas se aposentou por invalidez. Em função do número restrito de casos-piloto, não foram elencados dados como diagnóstico, tempo de afastamento, idade, sexo, cargo e setor, para preservação da identidade dos envolvidos.

Inicialmente, é válido ilustrar as dinâmicas das ações de RF, com base nos critérios apresentados no Quadro 2, por meio da descrição detalhada de um dos casos-piloto (servidor 1), que possuía laudo restritivo em função de adoecimento de natureza osteomuscular e cujo encaminhamento se enquadra nos critérios $\mathrm{C} 1$ e C2. O processo de RF foi realizado no período de 90 dias, conduzido por dois técnicos de referência, sendo o caso discutido regularmente com toda a equipe. Foram feitas 10 visitas ao posto de trabalho, totalizando 12,4 horas de avaliações e intervenções in loco em que se realizou observação da atividade de trabalho (I-1; I-2) e treinamento (I-2; I-4) para estimular as habilidades laborais, as capacidades remanescentes e o desempenho do servidor, respeitando-se as restrições laborais (O1; O2; O5). Ele também foi orientado acerca de seu processo saúde-doença, de práticas de autocuidado, prevenção de agravos e manejo de suas limitações funcionais $(\mathrm{O} 1$; O7). Estabeleceu-se uma comunicação regular e acolhedora entre a equipe, o servidor, seu colega de trabalho, chefia e demais envolvidos (O7; I-4; I-5) para: apresentar a metodologia do acompanhamento e seu objetivo; orientar a chefia imediata e o servidor acerca de suas responsabilidades durante o período de RF (O1; O3); discutir sobre as atividades compatíveis com a capacidade laborativa do servidor e a forma de cumprimento das restrições laborais (O2). Assim, após o término da licença médica, as atividades de trabalho foram definidas com o servidor, a chefia e colegas de setor. Essas foram gradualmente desempenhadas pelo servidor, respeitando as restrições laborais, por meio do treino de habilidades, adaptações em ferramentas de trabalho e orientações ergonômicas. Algumas tarefas passaram a ser executadas de maneira modificada, envolvendo a realização de pausas, tempo maior de execução e a troca de dominância para manuseio de algumas ferramentas (I-2; I-3; I-4). Os contatos com a chefia e com outros setores que se fizeram necessários foram realizados, sempre que possível, com o suporte do setor de RH (I-5). No decurso da RF, foi constatada melhora progressiva do desempenho do servidor diante da dinâmica e de demandas do seu local de trabalho, sendo sua adaptação ao trabalho considerada satisfatória pelos envolvidos ao final do acompanhamento. $\mathrm{O}$ caso foi monitorado por seis meses, no intuito de acompanhar longitudinalmente a adaptação do servidor ao trabalho que decorreu sem intercorrências. Por meio de relatos verbais, o servidor e a chefia avaliaram positivamente o trabalho feito pela equipe e consideraram que o acompanhamento favoreceu uma reinserção segura e produtiva do servidor.

Nas Tabelas 1 e $\mathbf{2}$ encontram-se, respectivamente, as quantidades dos procedimentos de intervenção e dos tipos de atendimentos realizados por especialidade.

A Tabela 3 apresenta informações sobre as intervenções realizadas nos casos-piloto e o número de dias de afastamento por LTS desses servidores um ano antes e um ano após a RF.

Tabela 1 Procedimentos de intervenção adotados para reabilitação funcional de oito trabalhadores de uma instituição federal de ensino superior

\begin{tabular}{lcc}
\hline Procedimentos & $n$ & $\%$ \\
\hline Intervenção individual & 29 & 28,4 \\
Contato telefônico & 24 & 23,5 \\
Visita ao ambiente de trabalho e outros procedimentos para análise da atividade de trabalho & 21 & 20,6 \\
Reunião externa (para fins de intervenção) & 14 & 13,7 \\
Intervenção com chefia & 7 & 6,9 \\
Mediação em saúde do trabalhador & 7 & 6,9 \\
Total & 102 & 100 \\
\hline
\end{tabular}

Tabela 2 Tipos de atendimentos por especialidade realizados na reabilitação funcional de oito trabalhadores de uma instituição federal de ensino superior

\begin{tabular}{lcc}
\hline Número de atendimentos & $n$ & $\%$ \\
\hline Psicologia & 33 & 32,4 \\
Terapia Ocupacional & 30 & 29,4 \\
Interdisciplinar" & 23 & 22,5 \\
Fisioterapia & 16 & 15,7 \\
Total & 102 & 100 \\
\hline
\end{tabular}

*Atendimento realizado por dois ou mais profissionais da equipe 
Tabela 3 Dados do primeiro ano das ações de reabilitação funcional de oito trabalhadores de uma instituição federal de ensino superior

\begin{tabular}{|c|c|c|c|c|c|c|c|c|}
\hline Servidor & $\begin{array}{l}\text { Predominância das } \\
\text { demandas de saúde }\end{array}$ & $\begin{array}{l}\text { Objetivos } \\
(0)^{* * *}\end{array}$ & 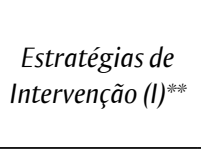 & $\begin{array}{c}\text { Tempo das } \\
\text { avaliações } \\
\text { e intervenções (l) } \\
\text { in loco } \\
\end{array}$ & $\begin{array}{l}\text { Duração das } \\
\text { Intervenções } \\
\text { casos-piloto }\end{array}$ & $\begin{array}{l}\text { Dias de } \\
\text { LTS* um } \\
\text { ano antes } \\
\text { da RF } \\
\end{array}$ & $\begin{array}{c}\text { Dias de } \\
\text { LTS* } \\
\text { um ano } \\
\text { após a RF }\end{array}$ & $\begin{array}{c}\% \text { de } \\
\text { redução }{ }^{e}\end{array}$ \\
\hline $1^{\mathrm{a}}$ & aspectos físicos & O1 a 03; 07 & 11 a 15 & 12,4 horas & 3 meses & 334 & 0 & $91 \%$ \\
\hline $2^{\mathrm{b}}$ & saúde mental & 01; 03 a 07 & $14 ; 15$ & 8 horas & 7 meses $^{\mathrm{d}}$ & 220 & 7 & $58 \%$ \\
\hline 3 & aspectos físicos & 01 a 03; 07 & 11 a 16 & 4,4 horas & 6 meses & 202 & 11 & $87 \%$ \\
\hline 4 & saúde mental & O1 a 07 & $11 ; 12 ; 14 ; 15$ & 2,4 horas & 4 meses & 141 & 0 & $39 \%$ \\
\hline 5 & aspectos físicos & $\mathrm{O} 1$ a 03; $\mathrm{O} 7$ & 11 a 16 & 6 horas & 5 meses & 62 & 0 & $17 \%$ \\
\hline 6 & aspectos físicos & 01 a 07 & 11 a 16 & 15 horas & 12 meses $^{\mathrm{d}}$ & 61 & 8 & $14 \%$ \\
\hline 7 & saúde mental & 01 a 07 & $11 ; 12 ; 14 ; 15$ & 2 horas & 5 meses $^{d}$ & 1 & 0 & $0 \%$ \\
\hline 8 & aspectos físicos & 01 a 03; 05 a 07 & 11 a 16 & 5 horas & 5 meses $^{d}$ & 0 & 29 & $-8 \%$ \\
\hline
\end{tabular}

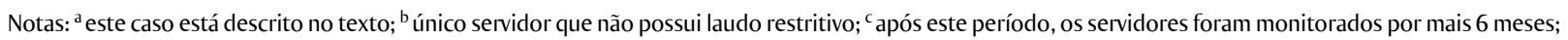

${ }^{\text {d }}$ casos não finalizados no ano de implantação da RF; e (número de dias de LTS 1 ano antes da RF - número de dias de LTS 1 ano após a RF)/365.

*Licença para tratamento de saúde; **** Descrição apresentada no Quadro 2.

Pode-se observar que os servidores 1 , 4 e 5 não se afastaram no ano subsequente ao trabalho de RF; os servidores 2, 6 e 11 se afastaram por período inferior a 12 dias; contabilizando-se $37 \%$ de redução do percentual médio do número de dias de afastamento do trabalho nos casos-piloto, quando comparados seus afastamentos antes e depois da RF. Destaca-se que esse resultado pode estar relacionado a fatores diversos, como adesão ao tratamento na RSS, melhora clínica, fatores pessoais do servidor e fatores ambientais do local de trabalho. É válido citar que o percentual de redução do número de dias de afastamento não se mostrou uma medida eficaz para analisar a evolução do servidor 7, cujas dificuldades para a reinserção não geraram afastamento do trabalho em nenhum momento. Ainda, esse indicador apresentou evolução negativa do servidor 8 , que esteve associada a episódios de piora de sua condição de saúde. Esses dois casos indicam a pertinência de agregar outros indicadores de resultado para acompanhamento das ações de RF.

A experiência da equipe permitiu identificar que o papel do técnico de referência da RF é atuar como facilitador do processo, buscando articulação e colaboração de todos os envolvidos. Diante da complexidade das variáveis que compõem uma situação real de trabalho, a equipe de RF oferece suporte técnico em saúde do trabalhador, não tendo governabilidade acerca da efetivação das proposições sugeridas durante a RF. O caráter das intervenções é eminentemente educativo, não se valendo de condutas impositivas ou punitivas. Entende-se que o determinante principal dos desfechos das ações de RF é o protagonismo dos envolvidos na situação real de trabalho.

\section{Reabilitação funcional: a interseção de saberes e práticas}

Aqui, retoma-se o desafio central da equipe de RF de atuar na interseção do processo saúde-doença com os fatores ligados à atividade de trabalho do servidor. Nesse cenário, desfechos bem-sucedidos podem abranger, por exemplo, situações em que: as restrições médicas sejam cumpridas; exista relacionamento respeitoso entre o trabalhador e seus colegas de setor; haja comunicação regular e acolhedora entre os trabalhadores que compõem a situação de trabalho e a equipe de RF; exista divisão de tarefas considerada adequada por todos os envolvidos; os trabalhadores validem coletivamente as mudanças necessárias para acolher as fragilidades de saúde de um colega.

Para buscar tais desfechos, é necessário o exercício constante de análise e articulação dos elementos que compõe essa complexa interseção das esferas da saúde e do trabalho. Na experiência relatada, a articulação dos pressupostos da EA e da ergologia à luz da CIF se mostrou profícua na condução das intervenções.

As intervenções de RF relatadas incluíram a compreensão dos processos de trabalho, de modo a considerar o trabalho e o trabalhador em atividade como elementos indispensáveis à análise. A análise da atividade é primordial para balizar definições que envolvem a saúde do servidor, sua atividade de trabalho e o contexto institucional. Nesse ponto, os referenciais da EA e da ergologia são pertinentes para compor as etapas do processo de RF que demandam a análise da atividade de trabalho do servidor.

A EA traz contribuições relevantes ao processo da RF ao abordar as dimensões prescrita e real do 
trabalho, e propor uma metodologia que busca compreender o trabalho para transformá-lo em prol da saúde dos trabalhadores, dentro dos limites considerados aceitáveis ${ }^{26,27,38}$. Ela congrega, dentre outros elementos, a análise e a reconfiguração da demanda, perpassando por etapas que envolvem a elaboração de hipóteses no decurso de observações sistemáticas das atividades e seus processos técnicos e reguladores, do ambiente de trabalho, das variabilidades envolvidas e do contexto institucional ${ }^{26,27,39}$. Sua metodologia está voltada para a definição de um diagnóstico local e global, que subsidia os processos de transformação.

Tais pressupostos orientaram a aproximação da equipe de RF da atividade de trabalho e do ambiente laboral dos servidores. A RF exige que sejam conjugadas variáveis de saúde, de segurança e de produtividade, pilares contemplados pela EA. As ferramentas da EA qualificam o estudo das nuances de cada situação de trabalho, permitindo reunir elementos substanciais, concretos e tangíveis com os quais se podem estabelecer relações entre a condição de saúde e a funcionalidade do servidor, bem como apropriar-se desse conhecimento da atividade e ambiente de trabalho para explorar possibilidades de transformação a partir do manejo de fatores ambientais. Nesse ponto, denota-se a pertinência da articulação com os pressupostos da CIF, que considera a funcionalidade e a incapacidade como resultado da interação entre as condições de saúde da pessoa e os fatores pessoais e ambientais.

Na perspectiva da CIF, os fatores ambientais se referem ao ambiente físico, social e de atitudes nos quais as pessoas vivem e conduzem sua vida, podendo atuar como barreiras ou facilitadores para a funcionalidade $^{36}$. O processo da RF se dedica ao estudo de fatores ambientais nos locais de trabalho, com o intuito de minimizar ou eliminar o impacto das barreiras e potencializar o efeito dos facilitadores na funcionalidade do servidor. Essa forma de atuar, por meio dos pressupostos da CIF, direcionou o processo de RF privilegiando a análise das capacidades e potencialidades do trabalhador, isto é, a equipe não se restringiu a considerar apenas elementos de restrições de saúde.

A articulação dos saberes das diferentes especialidades que compõem a equipe de RF, para responder às demandas de cada trabalhador e realidade de trabalho, denotou um claro exercício ergológico na tessitura de diretrizes para as práticas da RF. Isso porque a Ergologia pode ser entendida como uma filosofia em constante construção que busca abarcar o maior número possível de áreas do conhecimento para compreender o trabalho humano numa perspectiva transdisciplinar ${ }^{30,31}$.

Os principais elementos que impactaram transversalmente as ações de RF e que denotaram a pertinência da equipe assumir uma postura ergológica foram: (a) o debate de normas ${ }^{30-33,40}$, tais como as prerrogativas da atividade em si, da instituição empregadora e os parâmetros do próprio trabalhador; (b) a mobilização de valores dos atores envolvi$\operatorname{dos}^{30-33,40}$, tais como seus conceitos e preconceitos balizadores de condutas no trabalho que configuram processos de comunicação horizontais ou verticais, ações de cooperação ou competitividade, posturas de acolhimento e de exclusão; valores que permeiam as díades capacidade e incapacidade, produtividade ideal e real do trabalhador; e (c) o encontro de saberes dos diversos atores envolvidos ${ }^{30-33,40}$, incluindo trabalhador, gestores, colegas de trabalho, profissionais de RH e da equipe do SST. A postura ergológica no processo da RF favorece sua horizontalidade, de modo a legitimar e mobilizar debates considerando os valores e os múltiplos saberes que compõem o cenário das mediações e construções intrínsecas ao processo de RF.

Desse modo, é preciso contemplar todas as esferas do contexto de trabalho para não gerar práticas de individualização ou potenciais conflitos com os demais atores da situação de trabalho que configura o cenário das demandas de RF. A equipe segue com o propósito de avançar na consolidação das ações de RF na referida Ifes, dando continuidade a esse processo de construção laborioso, crítico e reflexivo, envolvendo esferas internas e externas ao SST. Assegura-se, assim, o constante aprimoramento da RF e o compromisso com a melhoria contínua dos serviços prestados ao servidor.

\section{Considerações finais}

A partir da experiência relatada neste trabalho, constata-se que os processos de adoecimento que acarretam mudanças na condição de saúde e funcionalidade de trabalhadores podem perfazer uma demanda para ações de RF. Equipes de RF colaboram na definição de quais atividades de trabalho são ou não indicadas ao trabalhador, respeitando sua condição de saúde e funcionalidade, os riscos do ambiente e/ou atividade e as demandas de produtividade do setor.

Ademais, entende-se que oferecer abordagens de natureza assistencial tipicamente utilizadas em serviços de saúde não configura ações de RF. A RF está circunscrita à atuação de profissional ou equipe de saúde do trabalhador de uma instituição e complementa os tratamentos ou terapêuticas que o servidor recorre na rede de saúde pública e/ou suplementar. A RF atua como um elo entre os cuidados de saúde e as demandas do contexto laboral e envolve, necessariamente, intervenções no contexto de trabalho. 
Para uma RF pautada em princípios mais bem definidos, levando-se em conta que o texto da legislação vigente é sucinto e só apresenta princípios gerais, seria importante a elaboração de diretrizes políticas, refletidas na legislação, que melhor definam o escopo conceitual e epistemológico da RF, assim como apresentem possibilidades teórico-metodológicas para viabilizar a implantação de serviços de RF nas Ifes. Espera-se que este relato de experiência possa contribuir para direcionar e definir uma matriz estrutural norteadora que permita a construção e consolidação de políticas de RF nas Ifes.

Por fim, para esta proposta, bem como para outras no campo da saúde e segurança no trabalho, enfatiza-se que os limitados reconhecimento e compreensão do impacto que as dimensões organizacionais, as condições e a organização do trabalho possuem nos processos de adoecimento e de afastamento dos trabalhadores comprometem a eficiência de estratégias de prevenção, promoção e recuperação da saúde.

\section{Contribuições de autoria}

Macedo DCF e Martins-Silva PO contribuíram igualmente no delineamento, na coleta e análise dos dados e na elaboração do manuscrito. Fantini AJE, Machado CYSB, Lima FO, Lacerda GA, Souza MAP, Toledo PR e Ribeiro SCS contribuíram na coleta e análise dos dados e na elaboração do manuscrito. Todos os autores aprovaram a versão final publicada e assumem responsabilidade pública pelo seu conteúdo.

\section{Agradecimentos}

Os autores agradecem o apoio e a colaboração intelectual de Regina Monteiro Campolina Barbosa, Catarina Nogueira Mota Coelho, Luciana Gonçalves de Oliveira Gotelipe e Gabriela Queiroz Vieira Neves no desenvolvimento deste trabalho.

\section{Referências}

1. Nunes AVL, Lins SLB. Servidores públicos federais: uma análise do prazer e sofrimento no trabalho. Rev Psicol Organ Trab. 2009;9(1):51-67.

2. Traesel ES, Merlo ARC. "Somos sobreviventes": vivências de servidores públicos de uma instituição de seguridade social diante dos novos modos de gestão e a precarização do trabalho na reforma gerencial do serviço público.

Cad Psicol Soc Trab. 2014;17(2):224-238.

3. Ribeiro CVS, Mancebo D. O servidor público no mundo do trabalho do século XXI. Psicol Cienc Prof. 2013;33(1):192-207.

4. Marques AL. Gerentes em organizações públicas: características do trabalho e contexto profissional. Gerais Rev Interinst Psicol. 2011;4(1):93-103.

5. Mattos CBM, Schlindwein VLDC. "Excelência e produtividade": novos imperativos de gestão no serviço público. Psicol Soc. 2015;27(2):322-331.

6. Schlindwein VLDC, Morais PR. Prevalência de transtornos mentais e comportamentais nas instituições públicas federais de Rondônia. Cad Psicol Soc Trab. 2014;17(1):117-127.

7. Oliveira LA, Baldaçara LR, Maia MZB. Afastamentos por transtornos mentais entre servidores públicos federais no Tocantins. Rev Bras Saude Ocup. 2015;40(132):156-169.
8. Serafim AC, Campos ICM, Cruz RM, Rabuske MM. Riscos psicossociais e incapacidade do servidor público: um estudo de caso. Psicol Cienc Prof. 2012;32(3):686-705.

9. Stradiotti JMM, Freire HBG, Souza JC, Rezende CL. Qualidade de vida e saúde geral dos servidores penitenciários do estado de Mato Grosso do Sul. Psicol Inf. 2014;18(18):47-70.

10. Vilas Boas AA, Morin EM. Qualidade de vida no trabalho: um modelo sistêmico de análise. Rev Adm Dial. 2017;19(2):62-90.

11. Vilas Boas AA, Pires AAS. Indicadores de qualidade de vida no trabalho de docentes de instituições federais de ensino superior das regióes Sudeste, Centro-Oeste e Distrito Federal. Braz Ap Sci Rev. 2018;2(1):19-51.

12. Brasil. Decreto ${ }^{\circ} 6.833$ de 29 de abril de 2009. Institui o Subsistema Integrado de Atenção à Saúde do Servidor Público Federal - SIASS e o Comitê Gestor de Atenção à Saúde do Servidor. Diário Oficial da União [internet]. 30 abr. 2009 [citado em 5 mar 2017]. Disponível em: http://www.planalto.gov.br/ ccivil_03/_ato2007-2010/2009/decreto/d6833.htm

13. Donato M, Zeitoune RCG. Reinserção do trabalhador alcoolista: percepção, limites e possibilidades de intervenção do enfermeiro do trabalho. Esc Anna Nery. 2006;10(3):399-407. 
14. Alevato H. Os desafios da reinserção laboral dos afastados por transtornos mentais e comportamentais. Rev Est Trab. 2011;V(9):2-24.

15. Cestari E, Carlotto MS. Reabilitação profissional: o que pensa o trabalhador sobre sua reinserção. Estud Pesq Psicol. 2012;12(1):93-115.

16. Brasil. Ministério do Planejamento, Orçamento e Gestão (MPOG). Portaria no 19 de 20 de abril de 2017. Aprova o anexo a esta Portaria, que dá nova redação ao Manual de Perícia Oficial em Saúde do Servidor Público Federal, sobre os procedimentos a serem observados quando da aplicação da Perícia Oficial em Saúde, de que trata a Lei $\mathrm{n}^{\circ}$ 8.112, de 11 de dezembro de 1990, e demais normas aplicadas à matéria. Diário Oficial da União [Internet]. 25 abr. 2017 [citado em 11 jan 2018]. Disponível em: http://pesquisa.in.gov.br/imprensa/jsp/visualiza/ index.jsp?data $=25 / 04 / 2017 \&$ jornal $=1 \&$ pagina $=54 \& \mathrm{t}$ otalArquivos $=84$

17. Brasil. Ministério do Planejamento, Orçamento e Gestão (MPOG). Portaria ${ }^{\circ} 1.261$ de 05 de maio de 2010. Institui os Princípios, Diretrizes e Ações em Saúde Mental que visam orientar os órgãos e entidades do Sistema de Pessoal Civil - Sipec da Administração Pública Federal sobre a saúde mental dos servidores. Diário Oficial da União [Internet]. 6 maio 2010 [citado em 11 jan 2018]. Disponível em: http://pesquisa.in.gov.br/imprensa/ $\mathrm{jsp} /$ visualiza/index.jsp?jornal $=1 \&$ pagina $=97 \& \mathrm{da}$ $\mathrm{ta}=06 / 05 / 2010$

18. Couto PR, Paschoal T. Relação entre ações de qualidade de vida no trabalho e bem-estar laboral. Psicol Argum. 2012;30(70):585-593.

19. Pinheiro FPHA, Silva GC, Taissuke ASN, Aquino CAB. Projeto ELABORar: uma experiência de intervenção junto a trabalhadores da Universidade Federal do Ceará. Rev Psicol. 2013;4(2):103-113.

20. Grande AJ, Silva V, Manzatto L, Rocha TBX, Martins GC, Vilela JGB. Determinantes da qualidade de vida no trabalho: ensaio clínico controlado e randomizado por clusters. Rev Bras Med Esporte. 2013;19(5):371-375.

21. Silva JAMG, Hotta TTH, Silva TH, Almeida MHM, Caromano FA. Desenvolvimento de um programa de promoção da saúde para trabalhadores administrativos. Saude Pesqui. 2017;10(3):557-566.

22. Brasil. Portal da Transparência. Brasília: Controladoria-Geral da União; 2017 [citado em 15 ago 2017]. Disponível em: http:// www.portaltransparencia.gov.br/paginainterna/603399-controle-social

23. Vivan CA. Acolhimento de servidores públicos readaptados: uma questão de saúde do trabalhador [trabalho de conclusão de curso]. São Paulo: Fundação Getúlio Vargas; 2014.

24. Santos JP. A escuta qualificada: instrumento facilitador no acolhimento ao servidor readaptado [trabalho de conclusão de curso]. São Paulo: Fundação Getúlio Vargas; 2014.
25. Maeno M, Vilela AGVR. Reabilitação profissional no Brasil: elementos para a construção de uma política pública. Rev Bras Saude Ocup. 2010;35(121):87-99.

26. Guérin F, Laville A, Daniellou F, Duraffourg J, Kerguelen A. Compreender o trabalho para transformá-lo: a prática da ergonomia. São Paulo: Blucher; 2001.

27. Ferreira LL. Sobre a análise ergonômica do trabalho. Rev Bras Saude Ocup. 2015;40(131):8-11.

28. Bendassoli PF, Soboll LAP. Clínicas do trabalho: filiações, premissas e desafios. Cad Psicol Soc Trab. 2011;14(1):59-72.

29. Consolino AMGAV, Salgado ECVC, Leão MABG. Interdisciplinaridade, saúde e trabalho: uma análise de publicações em bases científicas. Rev Cienc Hum Unitau. 2012;5(Spec No 1-2):190-210.

30. Schwartz Y, Duc M, Durrive L. O homem, o mercado e a cidade. In: Schwartz Y, Durrive L, organizadores. Trabalho \& Ergologia: conversas sobre a atividade humana. $2^{\mathrm{a}}$ ed. Niterói: EdUFF, 2010. p. 244-291.

31. Holz EB, Bianco MF. Ergologia: uma abordagem possível para os estudos organizacionais sobre trabalho. Cad EBAPE.BR. 2014;12(Spec No):494-512.

32. Trinquet, P. Trabalho e educação: o método ergológico. Rev HISTEDBR On-line. 2012;10(38e):93-113.

33. Schwartz Y, Durrive L. Glossário da ergologia. Laboreal. 2008;4(1), 23-28.

34. Brasil ACO. Promoção de saúde e a funcionalidade humana. Rev Bras Promoç Saude. 2013;26(1):1-4.

35. Organização Mundial da Saúde (OMS). CIF: Classificação Internacional de Funcionalidade, Incapacidade e Saúde. São Paulo: Edusp; 2003.

36. Organização Mundial da Saúde (OMS). Como usar a CIF: um manual prático para o uso da Classificação Internacional de Funcionalidade, Incapacidade e Saúde (CIF). Versão preliminar para discussão. Genebra: OMS; 2013.

37. Barbosa RMC. O processo de readaptação profissional em um hospital público: percepção dos trabalhadores de enfermagem portadores de doenças osteomusculares [dissertação]. Belo Horizonte: Universidade Federal de Minas Gerais; 2004.

38. Trombetta AP, Ramos FRS, Vargas MAO, Marques AMB. Experiências da equipe de centro de reabilitação: - o real do trabalho como questão ética. Esc Anna Nery. 2015;19(3):446-453.

39. Daniellou F, Béguin P. Metodologia da ação ergonômica: abordagens do trabalho real. In: Falzon P. Ergonomia. São Paulo: Blucher, 2007. p. 281-302.

40. Telles AL, Alvarez D. Interfaces ergonomiaergologia: discussão sobre trabalho prescrito e normas antecedentes. In: Figueiredo M, Athayde M, Brito J, Alvarez D, organizadores. Labirintos do trabalho: interrogações e olhares sobre o trabalho vivo. Rio de Janeiro: DP\&A; 2004. p. 63-89. 